\title{
Social discount rate: spaces for agreement
}

\author{
Takashi Hayashi ${ }^{1}$ - Michele Lombardi ${ }^{2,3}$
}

Received: 25 May 2021 / Accepted: 2 August 2021 / Published online: 17 August 2021

(C) The Author(s) 2021

\begin{abstract}
We study the problem of aggregating discounted utility preferences into a social discounted utility preference model. We use an axiom capturing a social responsibility of individuals' attitudes to time, called consensus Pareto. We show that this axiom can provide consistent foundations for welfare judgments. Moreover, in conjunction with the standard axioms of anonymity and continuity, consensus Pareto can help adjudicate some fundamental issues related to the choice of the social discount rate: the society selects the rate through a generalized median voter scheme.
\end{abstract}

Keywords Consensus Pareto - Social aggregation · Discounted utility model · Expected utility theory $\cdot$ Generalized median

JEL Classification D71 · D72 · D91

\section{Introduction}

We study the problem of aggregating stationary (exponential/geometric) discounted utility preferences into a social stationary discounted utility model. Given the dominant role of the representative agent model in macroeconomics, economic growth, environmental economics and many other fields, which is described with the stationary discounted utility theory, the problem of how we should construct such model in a consistent manner is a significant issue.

Takashi Hayashi

takashi.hayashi@glasgow.ac.uk

Michele Lombardi

m.lombardi@liverpool.ac.uk

1 Adam Smith Business School, University of Glasgow, Glasgow, UK

2 Management School, University of Liverpool, Liverpool, UK

3 DiSES, University of Napoli Federico II, Napoli, Italy 
The common practice requires the aggregation to satisfy the so-called Pareto principle. This principle requires that when each member of the society prefers one intertemporal allocation to another, the society's ranking must endorse this view.

Zuber (2011) and Jackson and Yariv (2015) provide a striking impossibility result that a social ranking satisfying the Pareto principle and the stationarity condition must be dictatorial, unless all the individuals have the same discount rate. ${ }^{1}$

Given that people have different attitudes to time, trade-offs must be made between the Pareto principle and stationarity to avoid dictatorial social rankings. In this study, we retain the stationarity requirement of social rankings and weaken the Pareto principle. $^{2}$ The reasons of this line of research are detailed below.

A non-dictatorial social ranking that is non-stationary is necessarily time inconsistent or time variant (Halevy 2015; Millner and Heal 2018). ${ }^{3}$ Time inconsistency is unacceptable because it prejudices the credibility of the social decision. Thus, the social decision must be time variant. However, this is not desirable either.

The reason is that a time variant social decision imposes no restriction on the dynamic process of the social welfare ranking, which makes the dynamic problem trivial. Indeed, time consistency is trivially satisfied by selecting any social ranking over consumption streams in day 1 and by committing to it from day 2 onwards. Such a time variant social decision may cause moral tensions. ${ }^{4}$

To illustrate this point, consider a two-individual society who must decide on how to divide resources between individuals. Suppose that individual A is patient and that individual B is totally myopic. ${ }^{5}$ In this situation, every social decision process that respects the Pareto principle must have the following form:

- Day 1: Everyone may obtain an amount of resources. Individual B will receive nothing tomorrow and onwards.

- From day 2 onwards: Because day 1 is over, individual B receives nothing.

Observe that the just described social decision process is dynamically consistent. Can the planner commit to such a time consistent social decision process? We suspect not, as it causes a significant moral tension of whether to allocate other resources to individual B from day 2 onwards. Time invariance can be viewed as a suitable restriction because it eases this tension.

Thus we propose a weakening of the Pareto principle, which we call consensus Pareto, and it requires the social preference to agree with the views of its members only when an intertemporal allocation gives a larger lifetime discounted utility than

\footnotetext{
1 They do not even need to assume time-additive preference. See (Koopmans 1960) and (Epstein 1983) for classes of stationary utilities that are not necessarily time-additive.

2 An alternative approach is taken by Chambers and Echenique (2018), who propose to weaken the completeness requirement on the social ranking.

3 In contrast to stationarity, which is a property of a single preference, time consistency is a consistency relationship between preferences at different histories. Stationarity and time consistency are equivalent when the relationship is time invariant; that is, when the ranking of future consumption streams is not affected by the shifting of preferences backwards or forwards in time.

4 These tensions could be dissolved by imposing some restrictions on the social decision process. Hayashi (2016) proposes a variant of such dynamic constraints.

5 Formally speaking, the case of total myopia is not in the class of stationary utilities. However, it can be taken to be as the limit of the class of stationary utilities.
} 
another for each of its members, according to every member's discount rate. This axiom is motivated by the fact that time discounting is not purely a matter of taste. Indeed, in calculating the lifetime discounted utility of each member of the society, the society should listen to the discounting opinions of all its members. While no single individual is responsible for his or her own time preference, the society as a whole is collectively responsible for its time preference.

Our proposal would remind the readers of the idea of collective responsibility proposed by by Gayer et al. (2014) in the context of belief heterogeneity, according to which an uncertain prospect Pareto-dominates another if the former gives a higher expected utility than the latter, for everyone and for all individuals' beliefs. ${ }^{6}$ Hence it will be helpful to explain the significance of our proposal by comparing it to the one in the context of belief heterogeneity. We have two reasons.

First, in the context of discount rate heterogeneity the conflict between the full Pareto condition and time consistency/invariance is necessary, while in the context of subjective uncertainty (Savage 1972) Pareto itself has no conflict with dynamic consistency/the sure-thing principle. Hence, even without a substantive motive for weakening Pareto, we have to, unless we have a strong reason to believe that we can maximize a time-variant objective with perfect commitment.

Second is that there is indeed a substantive motive to weaken Pareto in the context of aggregating time preferences. It is that that the formation of time preferences is affected by sociological aspects that go beyond the control of any individual.

Becker and Mulligan (1997) argue that an individual's time preference is endogenously determined by his/her parents' choice, which the individual cannot be responsible for. Also, empirical approach studies report that the time preference of an individual is related to his or her socioeconomic status (Lawrance 1991; Barsky et al. 1997; Tanaka et al. 2010; Dohmen et al. 2016). Although the precise causal effects are yet unclear, it is fair to say that acquiring and maintaining patience is not a straightforward process for which individuals can be kept fully accountable.

When individuals have heterogeneous discount factors, the Pareto principle requires that the society must endorse the view of its members when each of them prefers one intertemporal allocation to another. This endorsement must be made even when the superiority of one stream over another is merely justified by disparities in individuals' socioeconomic conditions for which individuals cannot be kept fully accountable. However, it seems bizarre to elevate these disparities to a social rank. This has motivated us to refine the Pareto principle by seeking "robustness" of the social welfare ordering to the distribution of individuals' socioeconomic characteristics, in some fashion.

In a recent paper, (Feng and Ke 2018) propose a Pareto criterion, called Intergenerational Pareto. This axiom treats present and future 'selves' of an individual as distinct individuals, whether their preferences are time-consistent or not, and apply the Pareto condition there. Although it is logically unrelated to our consensus Pareto, it shares the critique to the traditional view that an "integrated" individual is solely responsi-

\footnotetext{
6 One drawback of the Consensus Pareto argument, however, is that it takes preference representations as given, and consider utilities and beliefs separately as if they were primitive objects. Billot and Qu (2017) provide a set of consensus Pareto axioms expressed in terms of preference relations which can be used to restate our consensus Pareto in terms of preference relations.
} 
ble for his own time preference. Also, in a recent independent paper, Billot and Qu (2021) apply a version of the consensus Pareto axiom to analyze a problem about how much the social ranking can be closer to being time-consistent after aggregating rather time-inconsistent preferences in general. They also consider even weaker axioms, in the sense that the consensus Pareto condition is applied to restricted situations in which intertemporal trade-offs involve only limited number of periods, and obtain more permissive results for aggregation.

In contrast, we show that consensus Pareto has a sharp implication in aggregating stationary utilities into a stationary utility: the society's period utility function is a weighted average of individual utilities, which is along the lines of Harsanyi (1955), and the society's discount rate must be exactly equal to one individual's discount rate.

Although this result has a flavor of dictatorship under fixed profile, as it rules out any compromise in time discounting by means of averaging, it gives society the freedom to socially evaluate the discount rates of its members and to choose that which responds better to the society's view, under variable profiles. Indeed, by positing that the society's decision criterion satisfies two additional axioms-anonymity and continuity - , we provide the following complete characterization. The society's period utility function is the symmetric additive average of individual utilities, and the selection rule for the social discount rate has the form of the generalized median (Moulin 1980), which includes maximum, minimum and median as special cases.

Choosing the median of discount rates has been advocated by Millner and Heal (2014), and studied by Collective (2019) in view of strategy-proof voting. Our result can be seen as an axiomatic foundation of such political approach.

\section{The setting}

A set of $n$ agents, denoted by $N=\{1, \ldots, n\}$, must make a collective decision about sequences of social outcomes.

The agents are infinitely lived and consume in discrete periods $t \in\{1,2, \ldots\}$.

The set of pure social outcomes is $C$, which is assumed to be finite for simplicity. Following Harsanyi (1955), we consider the set of lotteries over the set $C$, denoted by $\mathcal{L}$, as the set of social outcomes per period. The set of sequences of lotteries is denoted by $\mathcal{L}^{\infty} \equiv \mathcal{L} \times \mathcal{L} \times \ldots$, which is endowed with the product metric.

We assume that agents' preference rankings and social decision criterion, which are defined over the set $\mathcal{L}^{\infty}$, follow the discounted utility theory. In other words, each agent's preference ranking is represented in the form

$$
\sum_{t} \beta_{i}^{t-1} u_{i}\left(\ell_{t}\right)
$$

where $\beta_{i} \in(0,1)$ is agent $i$ 's discount factor and $u_{i}: \mathcal{L} \rightarrow \mathbb{R}$ is given in the expected utility form ${ }^{7}$

\footnotetext{
7 When there is no danger of confusion, with some abuse of notation, sometimes we write $\ell$ for a lottery in a given period. 


$$
u_{i}(\ell)=\sum_{c \in C} \ell(c) v_{i}(c),
$$

where $v_{i}: C \rightarrow \mathbb{R}$ is agent $i$ 's (instantaneous) utility function. Similarly, the social decision criterion is represented in the form

$$
\sum_{t} \beta_{0}^{t-1} u_{0}\left(\ell_{t}\right)
$$

where $\beta_{0} \in(0,1)$ is the social discount factor and $u_{0}: \mathcal{L} \rightarrow \mathbb{R}$ is given in the expected utility form

$$
u_{0}(\ell)=\sum_{c \in C} \ell(c) v_{0}(c)
$$

Let $\mathcal{V}$ be the domain of decision criteria, with $\left(v_{i}, \beta_{i}\right)$ as a typical agent $i$ 's decision criterion. This domain is specified below.

A discounted utility aggregation rule $e^{8}$ maps a profile of individual decision criteria $\left(v_{i}, \beta_{i}\right)_{i \in N} \in(\mathcal{V} \times(0,1))^{n}$ into a social decision criterion $\left(v_{0}, \beta_{0}\right) \in \mathcal{V} \times(0,1)$

We present the following normalization condition, which is natural in our setting (see, for instance, Mongin 1995).

Minimal agreement and normalization: $\underline{c} \in C$ exists such that for all $v \in \mathcal{V}$ and all $c \in C$, it holds that

$$
v(c) \geq v(\underline{c}), \quad v(\underline{c})=0 \text { and } \sum_{c \in C} v(c)=1 .
$$

Consequently, $\mathcal{V}$ is a compact and convex subset of $\mathbb{R}^{|C|}$.

\section{Delegation of social discounting}

A well-known efficiency requirement for a social utility function is the so-called Pareto condition, which requires that if everyone agrees that one stream is (discernibly) superior to another, then the social utility function should exhibit the same preference.

Pareto principle: For all ', "' $\in \mathcal{L}^{\infty}$,

$$
\begin{gathered}
\sum_{t} \beta_{i}^{t-1} u_{i}\left(\ell_{t}\right)>\sum_{t} \beta_{i}^{t-1} u_{i}\left(\ell_{t}^{\prime}\right) \text { for all } i \in N \\
\Longrightarrow \sum_{t} \beta_{0}^{t-1} u_{0}\left(\ell_{t}\right)>\sum_{t} \beta_{0}^{t-1} u_{0}\left(\ell_{t}^{\prime}\right) .
\end{gathered}
$$

As explained in the introduction we propose a weakening of the Pareto Principle, which we call consensus Pareto, which requires that if everyone agrees that one stream is superior to another according to all the individuals' discount rates, then the social utility function should exhibit the same preference.

\footnotetext{
8 For expositional simplicity, we omit the full functional formulation and leave it implicit.
} 
Consensus Pareto: For all ' ${ }^{6}{ }^{\prime} \in \mathcal{L}^{\infty}$,

$$
\begin{gathered}
\sum_{t} \beta_{j}^{t-1} u_{i}\left(\ell_{t}\right)>\sum_{t} \beta_{j}^{t-1} u_{i}\left(\ell_{t}^{\prime}\right) \text { for all } i, j \in N \\
\Longrightarrow \sum_{t} \beta_{0}^{t-1} u_{0}\left(\ell_{t}\right)>\sum_{t} \beta_{0}^{t-1} u_{0}\left(\ell_{t}^{\prime}\right)
\end{gathered}
$$

We now state our first main result. If agents hold heterogeneous discount factors and their (instantaneous) utility functions are linearly independent, then any social decision criterion that respects consensus Pareto is dictatorial in the discount factor component: the social discount factor coincides with the discount factor of one of the agents. Moreover, the social utility can take only a limited form, namely, it is a linear combination of individual utility functions.

Theorem 1 Let $\left(\left(v_{i}, \beta_{i}\right)_{i \in N},\left(v_{0}, \beta_{0}\right)\right) \in(\mathcal{V} \times(0,1))^{n} \times(\mathcal{V} \times(0,1))$ be a profile of decision criteria for individuals and society, in which each criterion satisfies the minimal agreement and normalization. Suppose that $\beta_{1}, \cdots, \beta_{n}$ are all distinct and that $v_{1}, \cdots, v_{n}$ are linearly independent. Then, the social decision criterion $\left(v_{0}, \beta_{0}\right)$ satisfies consensus Pareto if and only if there is a non-zero vector $a \in \mathbb{R}_{+}^{n}$, with $\sum_{i \in N} a_{i}=1$, and an agent $s \in N$ such that

$$
v_{0}=\sum_{i \in N} a_{i} v_{i} \text { and } \beta_{0}=\beta_{s}
$$

Proof Let the premises hold. Since the proof of the "if" part of the statement is obvious, we prove the "only if" part. Suppose that $\left(v_{0}, \beta_{0}\right)$ satisfies consensus Pareto. Then, the Harsanyi theorem (Harsanyi 1955) shows that there is a non-zero matrix $\Gamma=$ $\left(\gamma_{i j}\right)_{i, j \in N} \in \mathbb{R}_{+}^{n^{2}}$ such that for all $\ell \in \mathcal{L}^{\infty}$,

$$
\sum_{t} \beta_{0}^{t-1} u_{0}\left(\ell_{t}\right)=\sum_{i \in N} \sum_{j \in N} \gamma_{i j} \sum_{t} \beta_{j}^{t-1} u_{i}\left(\ell_{t}\right)
$$

Restricting attention to sequences of the form $(\ell, \underline{c}, \underline{c}, \underline{c}, \cdots) \in \mathcal{L} \times C \times C \times \ldots$ yields

$$
u_{0}(\ell)=\sum_{i \in N} \sum_{j \in N} \gamma_{i j} u_{i}(\ell)
$$

By restricting attention to sequences in which an arbitrary $\ell \in \mathcal{L}$ appears in period $t$ and in which agents consume $\underline{c}$ in all other periods $t^{\prime} \neq t$, we obtain

$$
\beta_{0}^{t-1} u_{0}(\ell)=\sum_{i \in N} \sum_{j \in N} \gamma_{i j} \beta_{j}^{t-1} u_{i}(\ell) .
$$

Using (1) in (2), we obtain

$$
\beta_{0}^{t-1} \sum_{i \in N} \sum_{j \in N} \gamma_{i j} u_{i}(\ell)=\sum_{i \in N} \sum_{j \in N} \gamma_{i j} \beta_{j}^{t-1} u_{i}(\ell)
$$


for any arbitrary $\ell \in \mathcal{L}$. Since the individual instantaneous utility functions $v_{1}, \cdots, v_{n}$ are linearly independent, (3) yields

$$
\beta_{0}^{t-1} \sum_{j \in N} \gamma_{i j}=\sum_{j \in N} \gamma_{i j} \beta_{j}^{t-1}
$$

for all $i \in N$.

For $t=2$, (4) becomes

$$
\beta_{0} \sum_{j \in N} \gamma_{i j}=\sum_{j \in N} \gamma_{i j} \beta_{j}
$$

for all $i \in N$. By plugging (5) into (4), we obtain

$$
\left(\sum_{j \in N} \gamma_{i j} \beta_{j}\right)^{t-1}=\left(\sum_{j \in N} \gamma_{i j}\right)^{t-2} \sum_{j \in N} \gamma_{i j} \beta_{j}^{t-1}
$$

for all $i \in N$.

For $t=3$, (6) becomes

$$
\left(\sum_{j \in N} \gamma_{i j} \beta_{j}\right)^{2}=\left(\sum_{j \in N} \gamma_{i j}\right) \sum_{j \in N} \gamma_{i j} \beta_{j}^{2}
$$

for all $i \in N$.

Notice that the left-hand-side of (7) is

$$
\sum_{j \in N} \gamma_{i j}^{2} \beta_{j}^{2}+2 \sum_{j \in N} \sum_{k \in N-\{j\}} \gamma_{i j} \gamma_{i k} \beta_{j} \beta_{k}
$$

and the right-hand-side is

$$
\sum_{j \in N} \gamma_{i j}^{2} \beta_{j}^{2}+\sum_{j \in N} \sum_{k \in N-\{j\}} \gamma_{i j} \gamma_{i k}\left(\beta_{j}^{2}+\beta_{k}^{2}\right) .
$$

Hence simplifying (7) yields

$$
\sum_{j \in N} \sum_{k \in N-\{j\}} \gamma_{i j} \gamma_{i k}\left(\beta_{j}-\beta_{k}\right)^{2}=0
$$

for all $i \in N$. Since $\left(\beta_{1}, \ldots, \beta_{n}\right)$ are all distinct, we obtain

$$
\gamma_{i j} \gamma_{i k}=0
$$

for all $i, j \in N$ and $k \in N-\{j\}$. 
This means that every row of the matrix $\Gamma$ can have at most one non-zero entry. For any $i$ with a non-zero entry (there is at least one such $i$ since $\Gamma$ is a non-zero matrix), let $\gamma_{i j(i)}$ be such a non-zero entry. Then, (5) yields

$$
\beta_{0} \gamma_{i j(i)}=\gamma_{i j(i)} \beta_{j(i)}
$$

that is,

$$
\beta_{0}=\beta_{j(i)}
$$

for any such $i \in N$. Since $\left(\beta_{1}, \cdots, \beta_{n}\right)$ are all distinct, the only possibility is that $j(i)$ is identical for all $i$ who have the non-zero entry in $\left(\gamma_{i j}\right)_{j \in N}$. Let $s$ be such an index, so that $\beta_{0}=\beta_{s}$. Finally, let us define the non-zero vector $a$ as follows: $a_{i}=\gamma_{i s}$ if $i$ has a non-zero entry in $\left(\gamma_{i j}\right)_{j \in N}$; otherwise, $a_{i}=0$. Thus, we have that $v_{0}=\sum_{i \in N} a_{i} v_{i}$, where $\sum_{i \in N} a_{i}=1$ follows from the normalization condition. This completes the proof.

Our Theorem 1 may cover the existing impossibility result in the following form.

Corollary 1 (Zuber 2011 and Jackson and Yariv 2015) Let $\left(\left(v_{i}, \beta_{i}\right)_{i \in N},\left(v_{0}, \beta_{0}\right)\right) \in$ $(\mathcal{V} \times(0,1))^{n} \times(\mathcal{V} \times(0,1))$ be a profile of decision criteria for individuals and society, in which each criterion satisfies the minimal agreement and normalization. Suppose that $\beta_{1}, \cdots, \beta_{n}$ are all distinct and that $v_{1}, \cdots, v_{n}$ are linearly independent. Then, the social decision criterion $\left(v_{0}, \beta_{0}\right)$ satisfies Pareto principle if and only if there exists an agent $s \in N$ such that

$$
v_{0}=v_{s} \text { and } \beta_{0}=\beta_{s} .
$$

Proof There is an immediate violation of the Pareto principle if $a_{i}>0$ for some $i \neq s$.

\section{The social discounting selection rule}

Theorem 1 could be seen as a negative result, in the sense that it rules out any "averaging" method. However, the "discounting dictator" as shown in there is only for a fixed profile, and the theorem allows a class of reasonable selection rules in the variable-profile setting.

We now introduce two natural and well-known axioms that a social discounting selection rule may be required to satisfy-anonymity and continuity.

A social discounting selection rule $f$ for the set $\mathcal{V} \times(0,1)$ and the domain $(\mathcal{V} \times(0,1))^{n}$ is a function from $(\mathcal{V} \times(0,1))^{n}$ to the set of social decision criteria $\mathcal{V} \times(0,1)$.

An anonymous/impartial social discounting selection rule focuses on the individual decision criteria, and not on the identities of people who display particular decision criteria. 
Formally, suppose that $\sigma$ is a permutation of $N$. Such a permutation induces a map $\sigma$ on profiles of individual decision criteria:

$$
\begin{aligned}
\sigma & \left(\left(v_{1}, \beta_{1}\right),\left(v_{2}, \beta_{2}\right), \ldots,\left(v_{n}, \beta_{n}\right)\right) \\
\quad= & \left(\left(v_{\sigma(1)}, \beta_{\sigma(1)}\right),\left(v_{\sigma(2)}, \beta_{\sigma(2)}\right), \ldots,\left(v_{\sigma(n)}, \beta_{\sigma(n)}\right)\right) .
\end{aligned}
$$

Anonymity: A social discounting selection rule $f$ is anonymous if for every permutation $\sigma$ on $N$ and every profile of individual decision criteria $\left(\left(v_{1}, \beta_{1}\right)\right.$, $\left.\left(v_{2}, \beta_{2}\right), \ldots,\left(v_{n}, \beta_{n}\right)\right)$ in the domain of $f$, it holds that

$$
\begin{aligned}
f & \left(\left(v_{1}, \beta_{1}\right),\left(v_{2}, \beta_{2}\right), \ldots,\left(v_{n}, \beta_{n}\right)\right) \\
& =f\left(\left(v_{\sigma(1)}, \beta_{\sigma(1)}\right),\left(v_{\sigma(2)}, \beta_{\sigma(2)}\right), \ldots,\left(v_{\sigma(n)}, \beta_{\sigma(n)}\right)\right) .
\end{aligned}
$$

We now turn to the second axiom. A social discounting selection rule is continuous if changes in the social decision criterion can be bounded to be arbitrarily small by taking sufficiently small changes in individual decision criteria.

Formally, let $f^{-1}(A)$ be the set of all profiles of individual decision criteria for which $f\left(\left(v_{1}, \beta_{1}\right),\left(v_{2}, \beta_{2}\right), \ldots,\left(v_{n}, \beta_{n}\right)\right) \in A$.

Continuity: A social discounting selection rule $f$ is continuous (relative to the Euclidean topology) if for each open subset $A$ of $\mathcal{V} \times(0,1)$, the set $f^{-1}(A)$ is an open subset of $(\mathcal{V} \times(0,1))^{n}$.

A common objection to discontinuous social selection rules is sensitivity to small changes in individual decision criteria, and thus, to measurement errors. These issues are particularly relevant in empirical applications and policy debates, although they are possibly secondary in theoretical analyses (see Chichilnisky (Chichilnisky 1982, p. 346) for this argument).

To state the next theorem, given an integer $k \in\{1, \cdots, n\}$ and $n$ numbers $\left(\beta_{1}, \cdots, \beta_{n}\right) \in(0,1)^{n}$, let $k\left(\beta_{1}, \cdots, \beta_{n}\right)$ denote the $k$-th largest number out of $\left(\beta_{1}, \cdots, \beta_{n}\right)$. For example, when $k=1$ it refers to the largest number, when $k=n$ it refers to the smallest number. Also, when $n$ is odd and $k=\frac{n+1}{2}$ it refers to the median. In this sense we call the selection rule the generalized median rule (Moulin 1980 ).

Theorem 2 A social discounting selection rule satisfies consensus Pareto, anonymity and continuity if and only if there is an integer $k \in\{1, \cdots, n\}$ such that for all profiles $\left(\left(v_{i}, \beta_{i}\right)_{i \in N},\left(v_{0}, \beta_{0}\right)\right) \in(\mathcal{V} \times(0,1))^{n} \times(\mathcal{V} \times(0,1))$, where $\left(v_{0}, \beta_{0}\right)=$ $f\left(\left(v_{1}, \beta_{1}\right),\left(v_{2}, \beta_{2}\right), \ldots,\left(v_{n}, \beta_{n}\right)\right)$, it holds that

$$
v_{0}=\frac{1}{n} \sum_{i=1}^{n} v_{i}
$$

and

$$
\beta_{0}=k\left(\beta_{1}, \cdots, \beta_{n}\right) .
$$

Proof Let the premises hold. Since the proof of the "if" part of the statement is obvious, we prove the "only if" part. Suppose that $f$ satisfies consensus Pareto, anonymity 
and continuity. Let $\left(\left(v_{i}, \beta_{i}\right)_{i \in N},\left(v_{0}, \beta_{0}\right)\right) \in(\mathcal{V} \times(0,1))^{n} \times(\mathcal{V} \times(0,1))$ be a profile of decision criteria for individuals and society, in which each criterion satisfies the minimal agreement and normalization.

By anonymity, we assume without loss of generality that $\beta_{1} \leq \beta_{2} \leq \cdots \leq \beta_{n-1} \leq$ $\beta_{n}$. In addition, by continuity, we assume without loss of generality that $\beta_{1}<\beta_{2}<$ $\cdots<\beta_{n-1}<\beta_{n}$.

By restricting attention to sequences of the form $(\ell, \underline{c}, \underline{c}, \underline{c}, \cdots)$, where $\ell \in \mathcal{L}$ is an arbitrary lottery, Theorem 1 and anonymity implies that

$$
v_{0}=\frac{1}{n} \sum_{i=1}^{n} v_{i} .
$$

Now, we need only to show that (8) holds. To this end, we assume that agents' utilities $v_{1}, \ldots, v_{n}$ are linearly independent.

Suppose that $\beta_{0}=\beta_{s}$ for some $s \in N$. By continuity, it always holds that $\beta_{0}=\beta_{s}$. Hence, $k=n-s+1$, so that

$$
\beta_{0}=k\left(\beta_{1}, \cdots, \beta_{n}\right)
$$

To complete the proof, we need to show that the choice of $k$ is independent of the choice of individual utilities $v_{1}, \ldots v_{n}$. Without loss of generality, we know that all $\beta_{1}, \cdots, \beta_{n}$ are distinct. If the choice of $k$ depended on the profile $\left(v_{i}\right)_{i \in N}$, then there would be a jump of the social discount factor from some $\beta_{s}$ to another $\beta_{s^{\prime}}$, which would be a violation of continuity. This completes the proof. ${ }^{9}$

\section{Conclusions}

We have studied the problem of aggregating discounted utility preferences into a social discounted utility preference model. We use an axiom capturing a social responsibility of individuals' attitudes to time, called consensus Pareto.

We have shown that under any fixed profile, consensus Pareto implies generically that we have to select exactly one individual's discount rate, which rules out any averaging. We have shown, however, that the way of selecting such discount rate can

9 One may be puzzled by our continuity argument that only order statistics matter-because, otherwise, the selection rule jumps - and may have the impression that it is an artifact of the assumption that $N$ is finite. When there is a continuum of agents $i \in[0,1]$ and a profile of discount factors is given by a continuous (and monotone increasing/decreasing) function $\beta:[0,1] \rightarrow(0,1)$, the analogue of our argument of selecting $\beta_{0}$ from $\beta([0,1])$ allows $\beta^{-1}\left(\beta_{0}\right)$ to move continuously even when $\beta$ changes in a way that its ordinal nature is unchanged. Note, however, that the space of continuous functions is extremely small compared to the space of Lebesgue measurable functions, though it is the natural choice in the context of a continuum of agents. Moreover, the space of continuous functions does not allow studying anonymous selection rules, because continuity is not preserved under permutations (measure-preserving transformations).

When we consider the domain in which $\beta:[0,1] \rightarrow(0,1)$ is a Lebesgue measurable function, first we can use our argument in the subspace of simple functions defined over increasing families of subintervals, generated, for example, by binary expansions, and then we can extend it to the whole domain by continuity with respect to $L^{\infty}([0,1])$, because the domain consists of bounded functions. In this way, we obtain that the rule selects the social discount factor $\beta_{0}$ by means of a fixed percentile. 
be made reasonable across variable profiles. We showed that adding Anonymity and Continuity characterizes the generalized median rule.

Open Access This article is licensed under a Creative Commons Attribution 4.0 International License, which permits use, sharing, adaptation, distribution and reproduction in any medium or format, as long as you give appropriate credit to the original author(s) and the source, provide a link to the Creative Commons licence, and indicate if changes were made. The images or other third party material in this article are included in the article's Creative Commons licence, unless indicated otherwise in a credit line to the material. If material is not included in the article's Creative Commons licence and your intended use is not permitted by statutory regulation or exceeds the permitted use, you will need to obtain permission directly from the copyright holder. To view a copy of this licence, visit http://creativecommons.org/licenses/by/4.0/.

\section{References}

Barsky, R.B., Juster, F.T., Kimball, M.S., Shapiro, M.D.: Preference parameters and behavioral heterogeneity: An experimental approach in the health and retirement study. QJE 112, 537-579 (1997)

Becker, G.S., Mulligan, C.B.: The endogenous determination of time preference. Q. J. Econ. 112(3), 729758 (1997)

Billot, A., Qu, X.: Perfect Altruism Breeds Time Consistency, working paper (2021)

Billot, A., Qu, X.: Utilitarian Aggregation with Reasonably Heterogeneous Beliefs, mimeo (2017)

Chambers, C.P., Echenique, F.: On multiple discount rates. Econometrica 86, 1325-1346 (2018)

Chichilnisky, G.: Social aggregation rules and continuity. Q. J. Econ. 97, 337-52 (1982)

Collective, Geber A.: Preferences, Time. In: Laslier, J.F., Moulin, H., Sanver, M., Zwicker, W. (eds.) The Future of Economic Design. Studies in Economic Design. Springer, Cham (2019)

Dohmen, T., Enke, B., Falk, A., Huffman, D., Sunde, U.: Patience and the Wealth of Nations. Working Papers (2016) 12, Human Capital and Economic Opportunity Working Group

Epstein, L.G.: Stationary cardinal utility and optimal growth under uncertainty. J. Econ. Theory 31, 133-152 (1983)

Feng, T., Ke, S.: Social discounting and intergenerational Pareto. Econometrica 86, 1537-1567 (2018)

Gayer, G., Gilboa, I., Samuelson, L., Schmeidler, D.: Pareto Efficiency with Different Beliefs. J. Legal Stud. 43, 151-171 (2014)

Halevy, Y.: Time consistency: stationarity and time invariance. Econometrica 83, 335-352 (2015)

Harsanyi, J.C.: Cardinal welfare, individualistic ethics, and interpersonal comparisons of utility. J. Polit. Econ. 63, 309-321 (1955)

Hayashi, T.: Consistent updating of social welfare functions. Soc. Choice Welf. 46, 569-608 (2016)

Jackson, M.O., Yariv, L.: Collective dynamic choice: the necessity of time inconsistency. AEJ: Microeconomics 7.4, 150-78 (2015)

Koopmans, T.C.: Stationary ordinal utility and impatience. Econometrica , 287-309 (1960)

Lawrance, E.C.: Poverty and the rate of time preference: evidence from panel data. J. Polit. Econ. 99, 54-77 (1991)

Millner, A., Heal, G.: Resolving Intertemporal Conflicts: Economics vs Politics. NBER Working Paper No. 20705, (November 2014)

Millner, A., Heal, G.: Time consistency and time invariance in collective intertemporal choice. J. Econ. Theory 176, 158-169 (2018)

Mongin, P.: Consistent Bayesian Aggregation. J. Econ. Theory 66, 313-351 (1995)

Moulin, H.: On strategy-proofness and single peakedness. Public Choice 35, 437-455 (1980)

Savage, Leonard J.: The foundations of statistics. Courier Corporation (1972)

Tanaka, T., Camerer, C.F., Nguyen, Q.: Risk and time preferences: Linking experimental and household survey data from Vietnam. Am. Econ. Rev. 100, 557-71 (2010)

Zuber, S.: The aggregation of preferences: can we ignore the past? Theory Decis. 70, 367-384 (2011)

Publisher's Note Springer Nature remains neutral with regard to jurisdictional claims in published maps and institutional affiliations. 